\title{
RBBP7 is a prognostic biomarker in patients with esophageal squamous cell carcinoma
}

\author{
NING YU ${ }^{1}$, PENG ZHANG $^{2}$, LI WANG $^{3}$, XINJIA HE $^{4}$, SHANSHAN YANG $^{4}$ and HAIJUN LU ${ }^{4}$ \\ Departments of ${ }^{1}$ Ultrasonography and ${ }^{2}$ Gastroenterology, The Affiliated Hospital of Qingdao University; \\ ${ }^{3}$ Department of Nursing, The Affiliated Qingdao Hiser Hospital of Qingdao University; \\ ${ }^{4}$ Department of Oncology, The Affiliated Hospital of Qingdao University, Qingdao, Shandong 266000, P.R. China
}

Received April 14, 2018; Accepted September 20, 2018

DOI: $10.3892 / \mathrm{ol} .2018 .9543$

\begin{abstract}
Retinoblastoma-binding protein 7 (RBBP7) is an important component of several complexes that regulate chromatin metabolism. It is overexpressed in certain cancer types and serves conflicting roles in tumor progression. In the present study, the expression and roles of RBBP7 were explored in esophageal squamous cell carcinoma (ESCC). Immunohistochemical staining was used to detect RBBP7 expression in ESCC tissues. The mRNA sequencing profiles from the Cancer Genome Atlas and Genotype-Tissue Expression databases were mined to analyze the mRNA expression of RBBP7 in tissues. Proliferation, clone formation, apoptosis and Transwell invasion/migration assays were performed to explore the roles of RBBP7 in ESCC. RBBP7 was highly expressed in ESCC tissues. The protein and mRNA expression levels of RBBP7 were significantly elevated in tumor tissues compared with paired adjacent normal tissues. RBBP7 overexpression was associated with a poor overall survival in patients with ESCC. Furthermore, higher RBBP7 expression was significantly correlated with poor tumor differentiation, advanced regional lymph node involvement, and pathological TNM staging. Knockdown of RBBP7 in ESCC cells did not affect tumor apoptosis or tumor growth. However, the overexpression of RBBP7 significantly enhanced the invasion and migration of ESCC cells, whereas the knockdown of RBBP7 resulted in significantly decreased invasion and migration. The present study indicated that RBBP7 is a novel biomarker and prognosticator for patients with ESCC. Furthermore, RBBP7 serves crucial roles in promoting ESCC invasion and migration.
\end{abstract}

Correspondence to: Mr. Haijun Lu, Department of Oncology, The Affiliated Hospital of Qingdao University, 16 Jiang Su Road, Qingdao, Shandong 266000, P.R. China

E-mail: 1724912595@qq.com

Key words: retinoblastoma-binding protein 7, esophageal squamous cell carcinoma, prognosticator, invasion, migration

\section{Introduction}

Esophageal cancer is the eighth most common cause of cancer-related death in women and the fifth most common in men worldwide (1). Despite the elaborate diagnostic protocols and complex therapeutic strategies developed to date, the prognosis of patients with esophageal cancer remains unsatisfactory (2-4). The 5-year survival rate of patients with esophageal cancer is just $20-30 \%(5,6)$. A major issue is that the basic molecular mechanisms driving the development and progression of esophageal cancer are not fully understood. Until now, only a few tumor biomarkers or therapeutic targets have been identified for the clinical management of esophageal cancer. Hence, novel biomarkers and therapeutic targets are needed to improve the treatment of patients with esophageal cancer.

Retinoblastoma-binding protein 7 (RBBP7), also named retinoblastoma protein associated protein $46(\mathrm{RbAp} 46)$, is a ubiquitously expressed nuclear protein that is found in many histone deacetylase (HDAC) complexes, including the mSin3 co-repressor complex (7), the type B histone acetyltransferase complex (8), the core HDAC complex (9), the nucleosome remodeling and histone deacetylase (NuRD) complex (10), and the PRC2/EED-EZH2 complex (11), and the nucleosome remodeling factor complex (12). As a component of the mSin3 co-repressor complex, RBBP7 interacts directly with retinoblastoma protein and is involved in the transcriptional repression process (13). In the type $B$ histone acetyltransferase complex, RBBP7 is required for chromatin assembly following DNA replication (8). In the HDAC complex, RBBP7 promotes histone deacetylation and consequent transcriptional repression (8). In the NuRD complex, RBBP7 promotes transcriptional repression by histone deacetylation and nucleosome remodeling (10). RBBP7 specifically interacts with the BRCT domain of the BRCA1 tumor-suppressor protein and modulates its transcriptional activity to influence the regulation of cell proliferation and differentiation (14).

The roles of RBBP7 in cancer development are complicated. RBBP7 expression was found to be elevated in certain cancers including breast cancer and acute leukemias. In some kinds of tumors, the overexpression of RBBP7 increased stress-induced apoptosis and showed a growth inhibition activity (15-17). However, more recently, RBBP7 was found to induce the epithelial-mesenchymal transition and thereby contribute 
to cancer progression and metastasis $(18,19)$. Until now, the expression and roles of RBBP7 in esophageal squamous cell carcinoma (ESCC) have remained unclear. Here we investigated the expression, clinical significance, and functions of RBBP7 in ESCC. We identified that RBBP7 is a prognosticator for ESCC and plays vital roles in tumor migration and invasion.

\section{Materials and methods}

Clinical samples. We analyzed 126 formalin-fixed, paraffin-embedded (FFPE) ESCC specimens, of which 72 had adjacent non-tumor tissues, from patients undergoing resection at The Affiliated Hospital of Qingdao University (Qingdao, China), between April 2001 and June 2005. Retrospective clinicopathological data for these patients were also obtained, including age, sex, smoking history, alcohol consumption history, tumor size, lymph node involvement, tumor-node-metastasis (TNM) stage, and pathological grade (according to the 8th edition of the AJCC TNM staging guidelines). Overall survival (OS) was defined as the time from the date of surgery to the date of death. Disease-free survival (DFS) was defined as the time from the date of surgery to the date of recurrence or progression. The ages of these patients ranged from 39 to 95 years (average, 69 years). Eighty-seven patients had a history of smoking, and 72 patients had a history of alcohol consumption. The numbers of tissues staged as T1, T2, T3, and T4 were 10 (7.94\%), 15 (11.90\%), 86 (68.25\%), and $15(11.90 \%)$, respectively. The numbers of patients classified as G1, G2, and G3 were 22 (17.46\%), 51 (40.48\%), and 43 (34.13\%), respectively. Eighty patients had regional lymph node metastasis. The numbers of patients with stage I, II, and III disease were 26 (20.63\%), 40 (31.75\%), and 60 (47.62\%), respectively.

The use of these specimens and the patient information were approved by the Ethics Committee of The Affiliated Hospital of Qingdao University. All patients provided written consent for the use of their specimens and disease information for future investigations according to the guidance of the ethics committee.

Immunohistochemistry (IHC). IHC was used to detect RBBP7 expression in esophageal tumor tissues. In brief, esophageal tumor tissue was serially sectioned at $5-\mu \mathrm{m}$ thickness, deparaffinized in xylene, and rehydrated through a series of graded alcohol concentrations. The samples were boiled in a pressure cooker for $3 \mathrm{~min}$ to retrieve the antigens. Endogenous peroxidases were blocked using 3\% hydrogen peroxidase treatment for $10 \mathrm{~min}$. The sections were then incubated with anti-RBBP7 primary antibodies (20365-1-AP; Wuhan Sanying Biotechnology, Wuhan, China) overnight at $4^{\circ} \mathrm{C}$. Detection was performed with a peroxidase-conjugated secondary antibody at $37^{\circ} \mathrm{C}$ for $30 \mathrm{~min}$ and a diaminobenzidine (DAB) substrate kit was used to perform the chromogenic reaction. The sections were counterstained with hematoxylin. The slides were then dehydrated through graded alcohol concentrations and covered with coverslips. The staining intensity and percentage of RBBP7-positive tumor cells were assessed. The intensity of the staining was evaluated using the following criteria: 0 , negative; 1 , low; 2 , medium; and 3 , high. The extent of staining was scored as $0,0 \%$ stained; $1,1-25 \%$ stained; $2,26-50 \%$ stained; and 3, 51-100\% stained. The final scores were calculated by multiplying the scores of the intensity by those of the extent and dividing the samples into four grades: 0 , absent (-); 1-2, weak (+); 3-5, moderate $(++)$; and 6-9, strong $(+++)$.

Cell culture. Human ESCC cell lines TE1, KYSE30, and KYSE150 were bought from Creative Bioarray (New York, USA) and Eca109 was obtained from the Committee of Type Culture Collection of the Chinese Academy of Sciences (Shanghai, China). All cells were cultured with antibiotics at $37^{\circ} \mathrm{C}$ in a humidified $5 \% \mathrm{CO}_{2}$ incubator. Cells were cytogenetically tested and authenticated before freezing. All cell lines were cultured in Dulbecco's modified Eagle's medium (DMEM)/F-12K/RPMI-1640 medium supplemented with $10 \%$ fetal bovine serum (FBS).

Plasmid construction and stable cell line establishment. The complete coding sequence of the human $R B B P 7$ gene was cloned into vector pLV-EF1-MCS-IRES-Bsd (Biosettia, San Diego, CA, USA). Lentiviruses were produced in $293 \mathrm{~T}$ cells for the stable transfection of the cell lines, and an empty vector was used as the control. Tumor cells $\left(1 \times 10^{5}\right)$ were infected with $100 \mu \mathrm{l}$ of lentivirus supernatant $\left(1 \times 10^{10} \mathrm{TU} / \mathrm{ml}\right)$. Forty-eight hour later, blasticidin (InvivoGen, San Diego, CA, USA) was added for selection.

Short interfering RNA (shRNA) sequences were designed using Biosettia's shRNA designer (http://http://biosettia.com/support/shrna-designer/). Three recommended sequences for $R B B P 7$ were synthesized and cloned into the pLV-hU6-EF1 $\alpha$-puro vector (Biosettia). Scrambled sequences were used as controls. The lentiviruses were then produced in $293 \mathrm{~T}$ cells. The most efficient of the three stable cell lines was used for the relevant assays.

Western blot analysis. Whole-cell extracts were prepared by lysing cells with radioimmunoprecipitation assay (RIPA) lysis buffer with a proteinase inhibitor cocktail (Sigma-Aldrich; Merck KGaA, Darmstadt, Germany). Protein concentrations were quantified using a Pierce Protein Assay kit (Thermo Fisher Scientific, Inc., Waltham, MA, USA). Protein lysates (20 mg) were separated by SDS-PAGE, and target proteins were detected by western blot analysis with antibodies against RBBP7 (1:1,000), and $\beta$-actin $(1: 5,000)$ as a control.

Transwell assay. Migration and invasion assays were performed with $8.0-\mathrm{mm}$ pore inserts in a 24-well Transwell chamber. For this assay, $1 \times 10^{5}$ cells were isolated and added to the top chamber with DMEM/F-12K/RPMI-1640. The invasion assay was performed using 1/6 diluted Matrigel-coated filters (BD Biosciences, Franklin Lakes, NJ, USA). DMEM/ F-12K/RPMI-1640 with 10\% FBS was added to the bottom chamber and the cells were allowed to incubate for $24 \mathrm{~h}$. Cells that had migrated to the bottom of the filter were stained with a three-step staining system (Thermo Fisher Scientific, Inc.). All experiments were repeated independently at least three times.

Colony formation assay. The plate colony formation assay was performed by seeding 1,000 cells/well into 6-well plates in triplicate. Fresh whole medium was changed every 4 days. Two weeks after seeding, the medium was discarded and the cells were stained using $0.1 \%$ crystal violet in methanol (Sigma-Aldrich; Merck KGaA). 
A

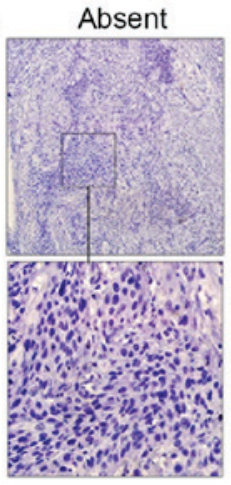

Weak

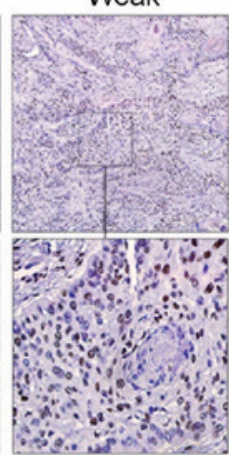

Moderate

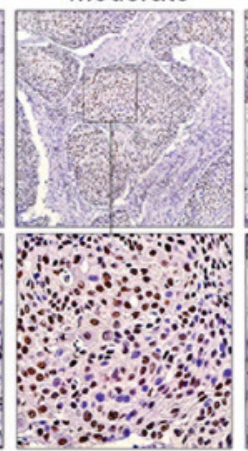

Strong

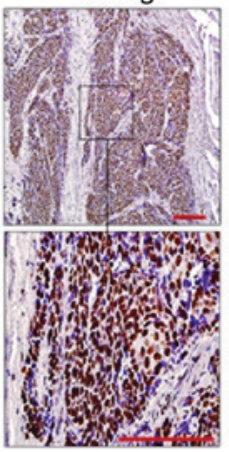

B

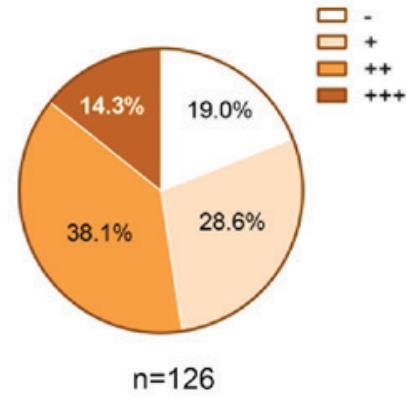

Figure 1. Expression pattern of RBBP7 in ESCC tissues. (A) Representative images of absent, weak, moderate and strong RBBP7 staining in ESCC tumor tissues. (B) The distribution of IHC results in 126 ESCC tumor tissues. Scale bar, $100 \mu \mathrm{m}$. RBBP7, retinoblastoma-binding protein 7; ESCC, esophageal squamous cell carcinoma; IHC, immunohistochemistry.

A

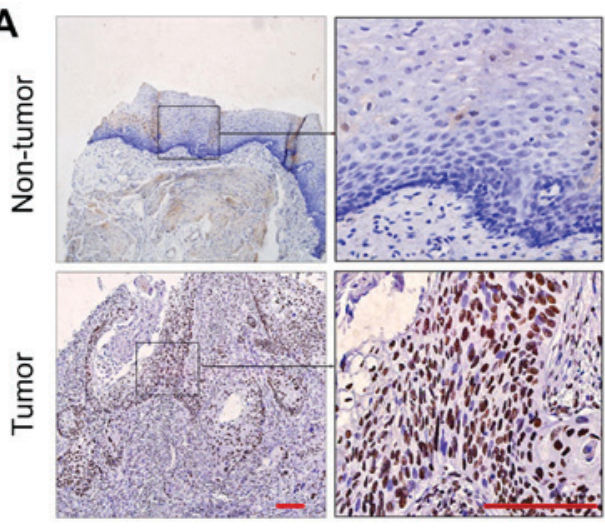

C

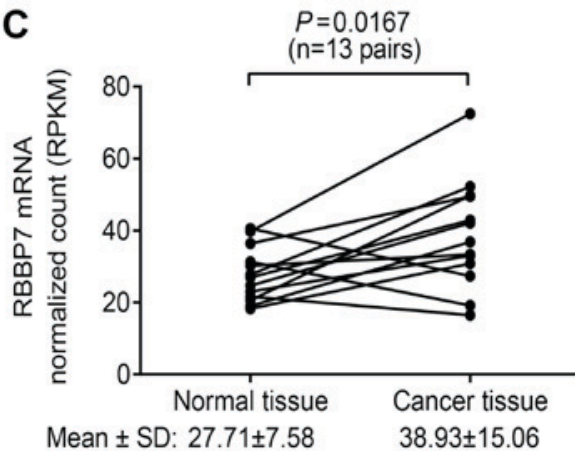

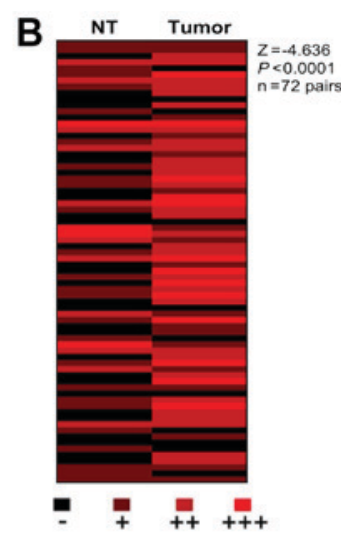

D

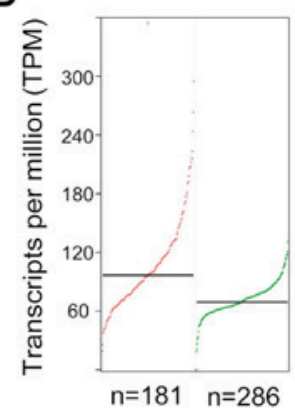

Figure 2. Expression of RBBP7 in ESCC tumor tissues and paired adjacent non-tumor tissues. (A) Representative images of RBBP7 staining in ESCC tumor tissues and paired NT tissues. (B) The differential expression levels of RBBP7 in 72 pairs of tumor and NT tissues are shown in a heat-map and were statistically analyzed using a Wilcoxon signed ranks test. (C) Thirteen pairs of esophageal cancer tumor tissues and NT tissues (from TCGA) were analyzed to compare the expression levels of RBBP7 mRNA. (D) Tumor tissues (181 from TCGA) and 286 normal tissues (from GTEx) were analyzed to compare the expression level of $R B B P 7 \mathrm{mRNA}$. Wilcoxon signed ranks test was used in (B); a paired t-test was used in (C). Scale bar, $100 \mu \mathrm{m}$. NT, non-tumor; RBBP7, retinoblastoma-binding protein 7; ESCC, esophageal squamous cell carcinoma; TGCA, The Cancer Genome Atlas; GTEx, Genotype-Tissue Expression.

Cell counting kit-8(CCK-8) cell viability assay. Two thousand cells per well were seeded into 96 -well plates and incubated. An aliquot of $10 \mu \mathrm{l}$ of CCK-8 was added to each well and incubated for $2 \mathrm{~h}$. The absorbance was measured at $450 \mathrm{~nm}$ to calculate the number of viable cells in each well. Each measurement was performed in triplicate and the experiments were repeated twice.

The cancer genome atlas (TGCA) data analysis. The TGCA data of 198 patients with esophageal cancer were downloaded from the TCGA website. Among them, 192 patients had complete RNA sequencing data. The Genotype-Tissue Expression (GTEx) data of 286 normal esophageal tissues were downloaded from the GTEx website. The normalized counts of the RNA expression of $R B B P 7$ were extracted and analyzed. OS and DFS were analyzed at TCGA.

Statistical analysis. Statistical analyses were performed using the IBM SPSS Statistics Program. The Wilcoxon signed rank test was used to compare the difference between tumor tissues and their paired adjacent non-tumor tissues. $\chi^{2}$ tests or Student's t-tests were used to analyze the association 
Table I. Association between RBBP7 expression and clinicopathological features of patients with ESCC.

\begin{tabular}{|c|c|c|c|c|c|}
\hline \multirow[b]{2}{*}{ Variable } & \multirow[b]{2}{*}{$\begin{array}{c}\text { Total } \\
(\mathrm{n}=126)\end{array}$} & \multicolumn{2}{|c|}{$R B B P 7$ level } & \multirow[b]{2}{*}{$\chi^{2}$} & \multirow[b]{2}{*}{ P-value } \\
\hline & & $\begin{array}{c}\text { Low } \\
(\mathrm{n}=70)\end{array}$ & $\begin{array}{l}\text { High } \\
(\mathrm{n}=54)\end{array}$ & & \\
\hline Sex & & & & 0.229 & 0.407 \\
\hline Male & 105 & 51 & 54 & & \\
\hline Female & 21 & 9 & 12 & & \\
\hline Age (years) & & & & 0.457 & 0.237 \\
\hline$<65$ & 45 & 19 & 26 & & \\
\hline$\geq 65$ & 81 & 41 & 40 & & \\
\hline Smoker & & & & 0.247 & 0.129 \\
\hline Yes & 87 & 38 & 49 & & \\
\hline No & 39 & 22 & 17 & & \\
\hline Alcoholic & & & & 0.719 & 0.388 \\
\hline Yes & 72 & 33 & 39 & & \\
\hline No & 54 & 27 & 27 & & \\
\hline T staging & & & & 0.379 & 0.238 \\
\hline $\mathrm{T} 1-\mathrm{T} 2$ & 25 & 14 & 11 & & \\
\hline $\mathrm{T} 3-\mathrm{T} 4$ & 101 & 46 & 55 & & \\
\hline Nodal involvement & & & & 6.544 & $0.016^{\mathrm{a}}$ \\
\hline Yes & 80 & 45 & 35 & & \\
\hline No & 46 & 15 & 31 & & \\
\hline Differentiation & & & & 5.936 & $0.023^{\mathrm{a}}$ \\
\hline G1-G2 & 83 & 46 & 37 & & \\
\hline G3 & 43 & 14 & 29 & & \\
\hline TNM staging & & & & 11.686 & $0.001^{\mathrm{a}}$ \\
\hline $\mathrm{I} / \mathrm{II}$ & 66 & 41 & 25 & & \\
\hline III & 60 & 19 & 41 & & \\
\hline
\end{tabular}

${ }^{\mathrm{a}} \mathrm{P}<0.05$ ( $\chi^{2}$ tests). RBBP7, retinoblastoma-binding protein 7; ESCC, esophageal squamous cell carcinoma; TNM, tumor-node-metastasis.

between RBBP7 expression and clinicopathological features. Kaplan-Meier curves were constructed and analyzed for the relevant variables. The log-rank test was used to analyze the differences in survival times among the patient subgroups. All probability values had a statistical power level of $90 \%$ and a 2 -sided level of $5 \%$. $\mathrm{P}<0.05$ was considered to indicate a statistically significant difference.

\section{Results}

$R B B P 7$ is overexpressed in ESCC. We classified a pattern of $\mathrm{RBBP} 7$ expression ranging from absent (scored as 0 ) to low, median, and high positive (scored as $1+, 2+$, and $3+$, respectively) (Fig. 1A). The results showed that RBBP7 was localized mainly in the cell nucleus and frequently overexpressed in ESCC. Among 126 ESCC specimens analyzed, the numbers of specimens scored as $0,1,2$, and 3 were 24 (19.0\%), 36 (28.6\%), 48 (38.1\%), and 18 (14.3\%) (Fig. 1B).

The differential expression of RBBP7 between ESCC tissues and normal esophageal tissues was statistically analyzed. As shown in Fig. 2A, RBBP7 was highly expressed in ESCC tissues as compared with the paired non-tumor tissues. We discovered that in 72 pairs of ESCC tissues and adjacent non-tumor tissues, RBBP7 protein was detected in the majority of the ESCC tissues but not in the non-tumor tissues. Statistical analysis confirmed that the abundance of RBBP7 protein in the ESCC tissues was significantly higher than that in the corresponding non-tumor tissues $(Z=-4.636$, $\mathrm{P}<0.0001$, Wilcoxon signed ranks test) (Fig. 2B).

Subsequently, we compared the mRNA expression of RBBP7 between esophageal carcinoma tissues and normal esophageal tissues. The whole-genome mRNA sequencing profiles of 13 paired esophageal carcinoma tissues and the corresponding normal tissues were downloaded from TCGA database. In concordance with our IHC results, the mRNA expression of RBBP7 was significantly elevated in esophageal carcinoma tissues as compared with paired non-tumor tissues (27.71 \pm 7.58 vs. $38.93 \pm 15.06$; $\mathrm{P}=0.0167$; paired t-test) (Fig. $2 \mathrm{C}$ ). Furthermore, we compared the mRNA expression of RBBP7 between esophageal carcinoma tissues from TCGA database $(n=181)$ and normal esophageal tissues from the GTEx database $(n=286)$. The results of this comparison also showed that the mRNA expression of RBBP7 was significantly higher in the esophageal carcinoma tissues ( $\mathrm{P}<0.05$; unpaired t-test) (Fig. 2D). 
Table II. Association between RBBP7 mRNA expression and clinicopathological features of patients with ESCA from TCGA.

\begin{tabular}{|c|c|c|c|c|}
\hline Variable & Total & $R B B P 7$ mRNA (RPKM) & $\mathrm{t}$ value & P-value \\
\hline Sex & & & -0.883 & 0.635 \\
\hline Male & 166 & $37.71 \pm 17.83$ & & \\
\hline Female & 31 & $41.03 \pm 17.04$ & & \\
\hline Age (years) & & & 0.607 & 0.148 \\
\hline$<65$ & 108 & $38.85 \pm 19.17$ & & \\
\hline$\geq 65$ & 76 & $37.23 \pm 15.49$ & & \\
\hline T staging & & & -1.152 & 0.259 \\
\hline $\mathrm{T} 1-\mathrm{T} 2$ & 73 & $35.24 \pm 14.57$ & & \\
\hline $\mathrm{T} 3-\mathrm{T} 4$ & 91 & $38.36 \pm 19.14$ & & \\
\hline Nodal staging & & & -2.477 & $0.024^{\mathrm{a}}$ \\
\hline N0 & 80 & $33.39 \pm 11.70$ & & \\
\hline $\mathrm{N} 1-\mathrm{N} 3$ & 84 & $39.96 \pm 20.43$ & & \\
\hline \multicolumn{5}{|l|}{ Differentiation } \\
\hline G1-G2 & 95 & $35.00 \pm 15.37$ & -2.153 & $0.035^{\mathrm{a}}$ \\
\hline G3 & 48 & $42.74 \pm 22.42$ & & \\
\hline TNM staging & & & -2.218 & $0.028^{\mathrm{a}}$ \\
\hline I-II & 96 & $33.87 \pm 13.97$ & & \\
\hline III & 64 & $39.74 \pm 20.61$ & & \\
\hline
\end{tabular}

Independent t-test; ${ }^{\mathrm{a}} \mathrm{P}<0.05$. ESCA, esophageal carcinoma; RBBP7, retinoblastoma-binding protein 7; TCGA, The Cancer Genome Atlas; TNM, tumor-node-metastasis.

RBBP7 is a biomarker of advanced nodal stage and TNM stage. To investigate the clinicopathological significance of RBBP7 in ESCC, we analyzed the potential correlations between RBBP7 expression and the clinicopathological characteristics of ESCC patients. As shown in Table I, a high expression of RBBP7 was positively correlated with poor differentiation $\left(\chi^{2}=5.936, \mathrm{P}=0.023\right.$, Chi-squared test $)$, regional lymph node involvement $\left(\chi^{2}=6.544, \mathrm{P}=0.016\right.$, Chi-squared test $)$, and advanced pTNM staging $\left(\chi^{2}=11.686, \mathrm{P}=0.001\right.$, Chi-squared test). No significant correlations were observed between RBBP7 expression and sex, age, smoking history, alcohol consumption history, or T staging.

Subsequently we analyzed the mRNA expression of RBBP7 and the corresponding clinical data of esophageal carcinoma patients from TCGA database. We found that a high mRNA expression of RBBP7 was significantly correlated with poor differentiation $(\mathrm{t}=-2.153, \mathrm{P}=0.035$, unpaired $\mathrm{t}$-test), regional lymph node involvement ( $\mathrm{t}=-2.477, \mathrm{P}=0.024$, unpaired $\mathrm{t}$-test), and advanced pTNM staging $(\mathrm{t}=-2.218, \mathrm{P}=0.028$, unpaired t-test) (Table II).

RBBP7 is a prognosticator of poor survival in ESCC patients. We then performed a Kaplan-Meier survival analysis to evaluate whether RBBP7 expression in ESCC tissues could be used as a prognostic factor for ESCC patients. As shown in Fig. 3A and B, the median OS and DFS of ESCC patients with a higher expression of RBBP7 were significantly shorter than those of patients with a lower expression of RBBP7 (1,756 vs. 2,307 days and 1,380 vs. 1,980 days, respectively, log-rank test).
Next, we analyzed the mRNA expression of RBBP7 and the corresponding follow-up data of esophageal carcinoma patients from TCGA database to assess the prognostic significance of RBBP7 mRNA expression. In concordance with the immunohistochemical analysis, the median OS and DFS of patients with a higher mRNA expression of RBBP7 were significantly poorer than those of patients with a lower mRNA expression of RBBP7 (19.71 vs. 32.42 months and 13.01 vs. 24.05 months, respectively; log-rank test) (Fig. $3 \mathrm{C}$ and D).

$R B B P 7$ promoted the migration and invasion of ESCC cells. To explore the roles of RBBP7 in tumor progression, we established stable cell lines in which RBBP7 was overexpressed or knocked down. RBBP7 was knocked down in two ESCC cell lines (TE1 and Eca109) (Fig. 4A) and overexpressed in two other ESCC cell lines (KYSE30 and KYSE150) (Fig. 4B). The roles of RBBP7 in tumor growth, survival, migration, and migration were explored in vitro. The results of CCK-8 assays showed that RBBP7 knockdown had no effect on tumor viability (Fig. 5A). Colony formation assays showed that RBBP7 inhibition did not change the colony formation rates (TE1: $21.0 \pm 1.7$ vs. $23.5 \pm 0.87 ; \mathrm{P}=0.266$; Eca109: $18.4 \pm 0.95$ vs. $19.0 \pm 0.58 ; \mathrm{P}=0.619$; unpaired t-tests) (Fig. $4 \mathrm{C}$ ). RBBP7 knockdown did not change the rate of apoptosis under normal culture conditions (TE1: $5.4 \pm 0.4$ vs. $5.3 \pm 0.2 ; \mathrm{P}=0.231$; Eca109: $4.1 \pm 0.2$ vs. $5.2 \pm 0.3 ; \mathrm{P}=0.352$; unpaired t-tests) (Fig. 4D). These data suggested that RBBP7 has no roles in maintaining ESCC cell growth and survival.

However, the changes in RBBP7 expression showed significant effects in the cell migration and invasion assays. 
A

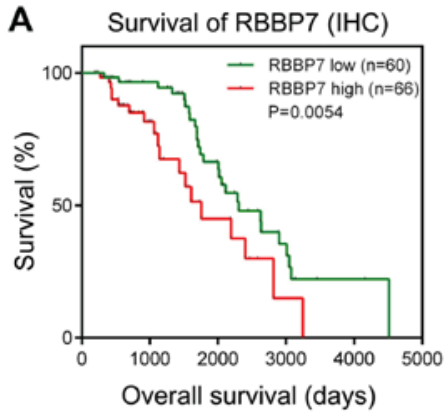

C Survival of RBBP7 (TCGA)

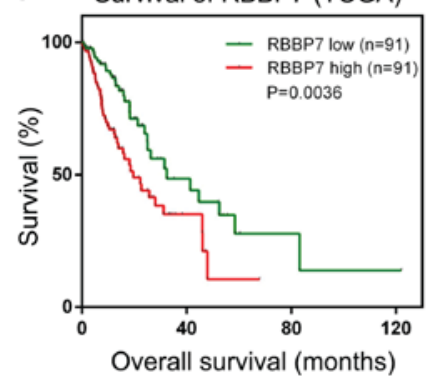

B Survival of RBBP7 (IHC)

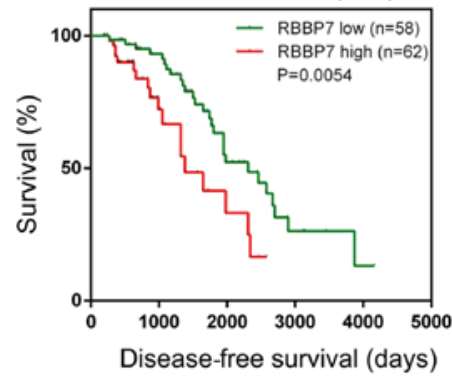

D Survival of RBBP7 (TCGA)

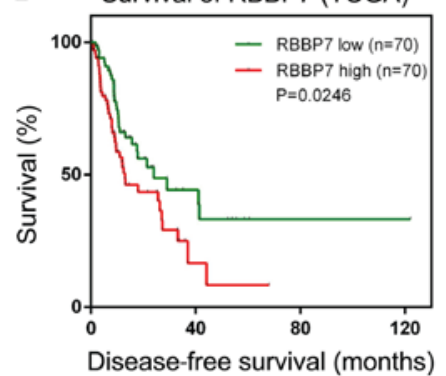

Figure 3. Clinical significance of RBBP7 in ESCC. (A and B) Kaplan-Meier analysis of overall survival of the 126 patients with ESCC according to different RBBP7 levels. (C and D) Kaplan-Meier analysis of overall survival and disease-free survival of the 182 patients with esophageal cancer according to different RBBP7 mRNA levels. Log-rank tests were used in (A-D). RBBP7, retinoblastoma-binding protein 7; ESCC, esophageal squamous cell carcinoma; IHC, immunohistochemistry; TCGA, The Cancer Genome Atlas.
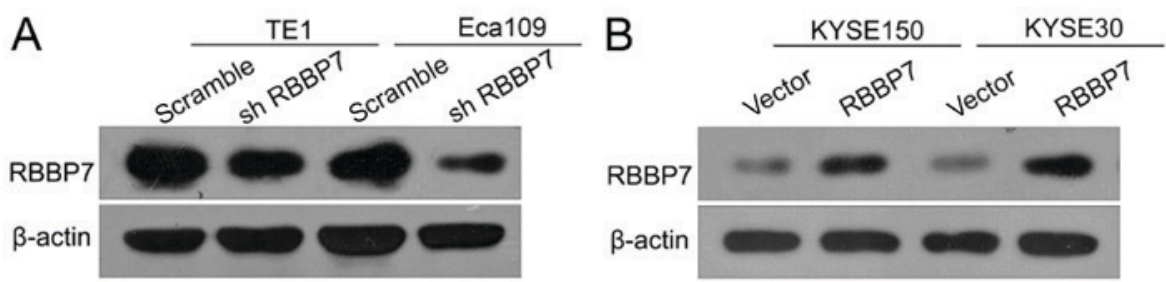

C
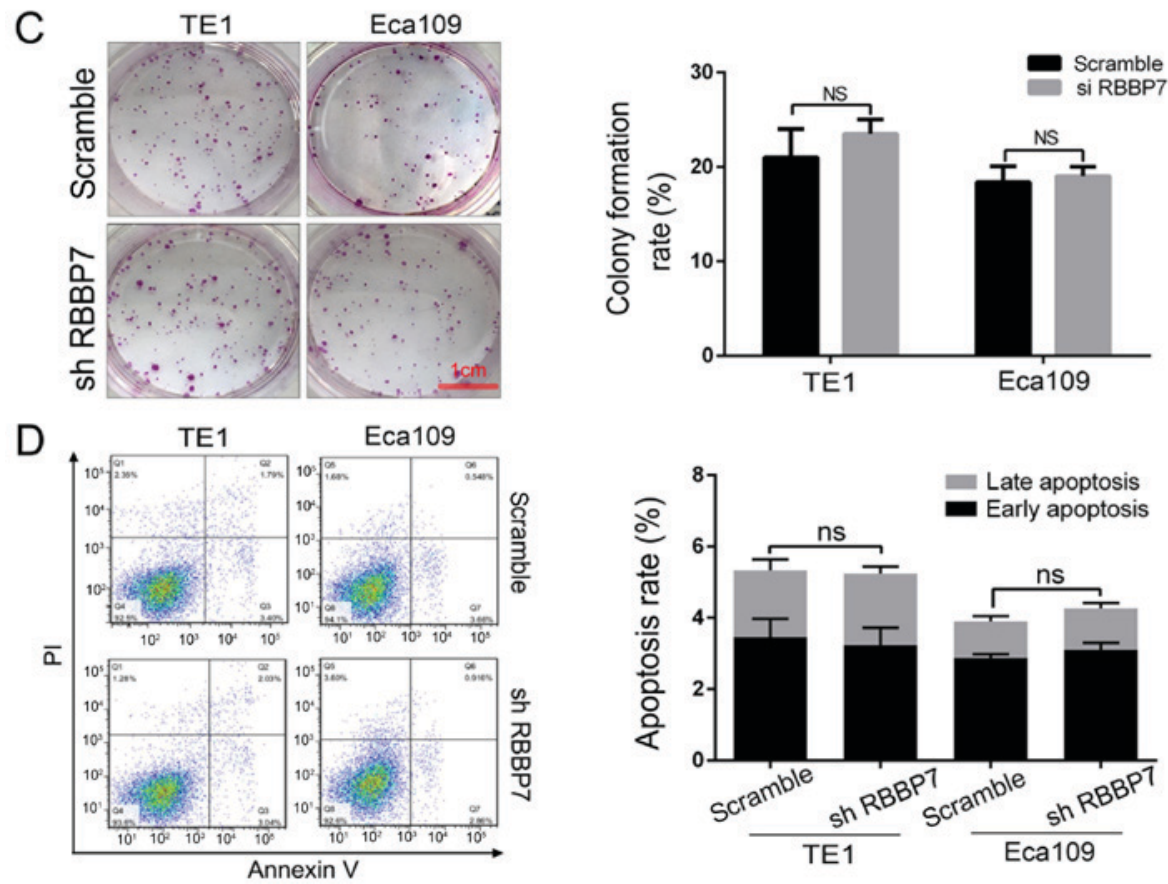

Figure 4. RBBP7 has no effect on tumor growth and survival processes in ESCC. (A) TE1 and Eca109 cells were transfected with lentiviruses to knock down $R B B P 7$ expression. Western blot assays were performed to verify the stable cell lines. (B) KYSE150 and YSE30 cells were transfected with lentiviruses to upregulate RBBP7 expression. Western blot assays were performed to verify the stable cell lines. (C) A plate colony formation assay was used to detect the colony formation rate. (D) Flow cytometry analysis was used to detect the cell apoptosis rate. Unpaired $t$ tests were used in (C) and (D); data are shown as the mean values \pm standard deviation. Scale bar, $1 \mathrm{~cm}$. RBBP7, retinoblastoma-binding protein 7; ESCC, esophageal squamous cell carcinoma; ns, not significant; PI, propidium iodide. 

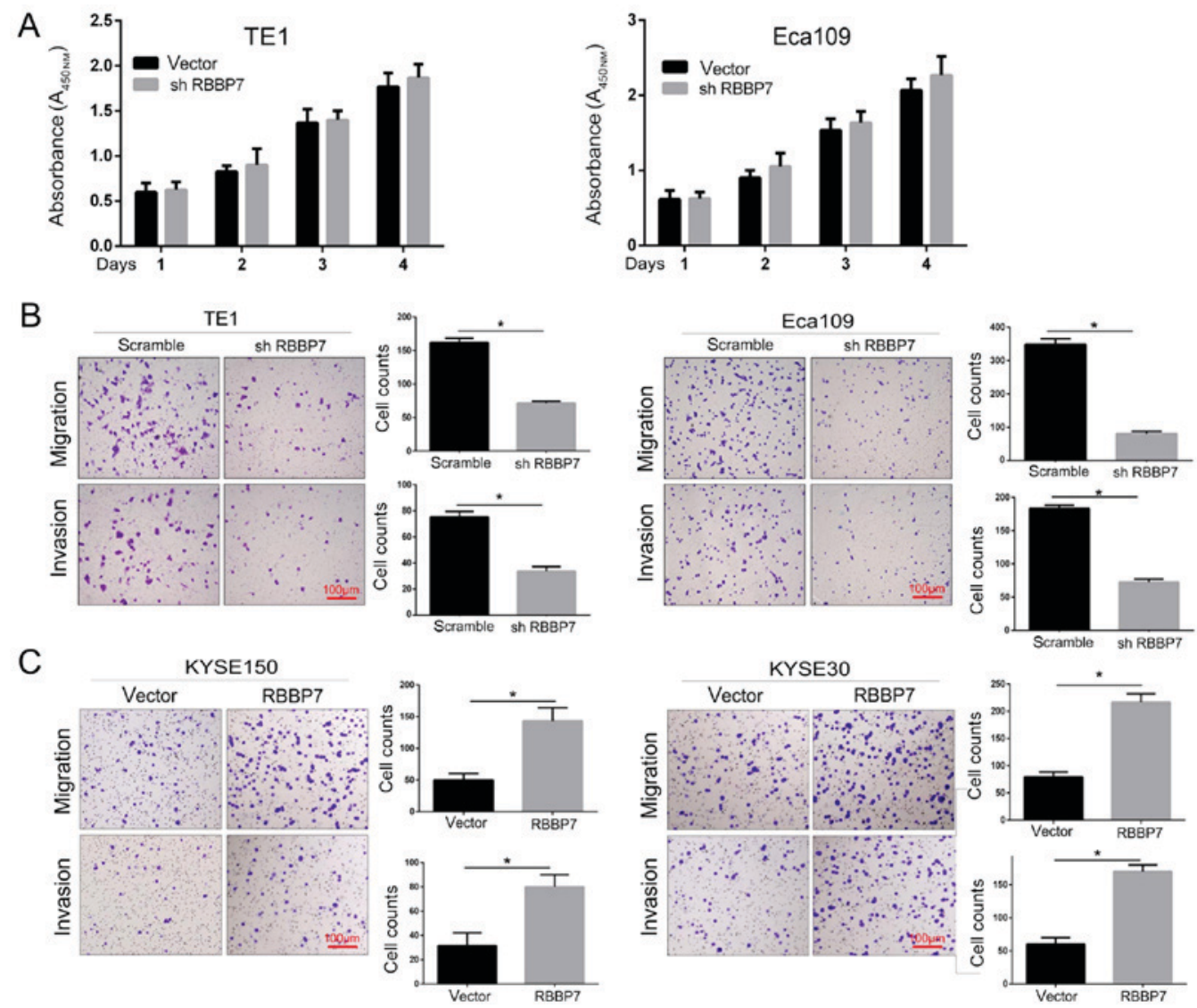

Figure 5. RBBP7 promotes ESCC cell migration and invasion. (A) The CCK-8 assay was used to detect the cell viability. (B and C) Transwell migration (upper panels) and Matrigel invasion (lower panels) assays were used to detect the migratory and invasive capacities of ESCC cells. Quantification of migrated cells and invaded cells through the membrane is shown. The experiments were performed independently three times. "P<0.05; scale bar, $100 \mu \mathrm{m}$. RBBP7, retinoblastoma-binding protein 7; ESCC, esophageal squamous cell carcinoma; CCK-8, cell counting kit-8.

As shown in Fig. 5B and C, RBBP7 knockdown significantly inhibited the migration and invasion of TE1 and Eca109 cells. Conversely, RBBP7 overexpression strongly enhanced the migration and invasion of KYSE150 and KYSE30 cells. Taken together, these results suggested that RBBP7 has vital roles in the migration and invasion of ESCC cells.

\section{Discussion}

To the best of our knowledge, this is the first study to have investigated the expression, clinical significance, and roles of RBBP7 in ESCC. We found that RBBP7 was frequently overexpressed in ESCC tissues. A higher RBBP7 expression was significantly correlated with a poorer prognosis. This suggests that RBBP7 might be a cancer-related protein with a functional role in tumor progression.

RBBP7 was reported to function as a tumor-suppressor gene that inhibits tumor growth by mediating JNK signaling (15) or binding to HDAC1 and Sp1 (19). RBBP7 also facilitated stress-induced apoptosis and suppressed tumorigenicity in breast epithelial cells (16). Nevertheless, we found that RBBP7 had no effect on tumor growth and survival in ESCC. Furthermore, the overexpression of RBBP7 in ESCC tissues at both the protein and mRNA levels correlated with poorer differentiation, regional lymph node involvement, and advanced pTNM staging, suggesting that RBBP7 might promote tumor progression in patients with
ESCC. We inferred that RBBP7 might have complex roles that may vary among different kinds of tumors owing to the tissue-specificity and intricate functions of the HDAC complexes that RBBP7 interacts with. This hypothesis is well supported by several published studies. In a recent study, RBBP7 overexpression was found to facilitate the epithelial-mesenchymal transition and promote migration and invasion (18). RBBP7 is a component of the Mi2/NuRD complex. Additionally, RBBP7 could be recruited by TWIST to the proximal regions of the E-cadherin promoter to repress the transcription of E-cadherin and thereby promote cell migration and invasion (20). Another report revealed that RBBP7 can be recruited to the Sp1 site of the RECK promoter region by Sp1 to activate RECK, leading to increased MMP9 activity that may promote metastasis (19). These studies have suggested that RBBP7 might play important roles in the epithelial-mesenchymal transition and contribute to cancer metastasis, providing a molecular mechanism for our findings that RBBP7 promoted the migration and invasion of ESCC cells in vitro. This potential functional role of RBBP7 in ESCC progression was further supported by our clinical analysis results, which showed that a high RBBP7 expression is significantly correlated with advanced regional lymph node involvement and pTNM staging. Together, these results suggested that RBBP7 may facilitate the metastasis of ESCC. Further studies are needed to verify this hypothesis and clarify the underlying mechanisms. 
We found that the OS and DFS of patients with ESCC who had a higher expression of RBBP7 were significantly shorter than those of patients who had a lower expression of RBBP7. This might be the consequence of a higher metastasis risk in ESCC patients with higher RBBP7 expression levels. Our findings suggested that a high expression of RBBP7 could be used as a prognosticator for poor survival. Prospective clinical trials are needed to verify the prognostic roles and usefulness of RBBP7 in ESCC.

Some limitations of this study are noted. Because nearly all the patients with esophageal carcinoma in China have ESCC, we did not include the clinical data of esophageal adenocarcinoma in this study. As a result, the expression and clinical significance of RBBP7 in esophageal adenocarcinoma remain unclear.

In conclusion, our findings demonstrated that RBBP7 was highly expressed in esophageal cancer. RBBP7 promoted esophageal cancer cell migration and invasion. We identified RBBP7 as a prognostic biomarker for patients with esophageal carcinoma.

\section{Acknowledgements}

Not applicable.

\section{Funding}

The present study was supported by the Key Program of Natural Science Foundation of Shangdong (grant no. 2017YJ0201).

\section{Availability of data and materials}

The datasets used and/or analyzed during the current study are available from the corresponding author on reasonable request.

\section{Authors' contributions}

Study concept and design: HL and NY. Acquisition of data: NY. Analysis and interpretation of data: PZ. Drafting of the manuscript: NY. Critical revision of the manuscript for important intellectual content: HL. Statistical analysis: LW, XH and SY. Study supervision: HL.

\section{Ethics approval and consent to participate}

The use of these specimens and the patient information were approved by the Ethics Committee of The Affiliated Hospital of Qingdao University.

\section{Patient consent for publication}

All patients provided written consent for the use of their specimens and disease information for future investigations according to the guidance of the ethics committee.

\section{Competing interests}

The authors declare that they have no competing interests.

\section{References}

1. Enzinger PC and Mayer RJ: Esophageal cancer. N Engl J Med 349: 2241-2252, 2003.
2. Kleinberg L and Forastiere AA: Chemoradiation in the management of esophageal cancer. J Clin Oncol 25: 4110-4117, 2007.

3. Wouters MW, Karim-Kos HE, le Cessie S, Wijnhoven BP, Stassen LP, Steup WH, Tilanus HW and Tollenaar RA: Centralization of esophageal cancer surgery: Does it improve clinical outcome? Ann Surg Oncol 16: 1789-1798, 2009.

4. Brücher BL, Swisher SG, Königsrainer A, Zieker D, Hartmann J, Stein H, Kitagawa Y, Law S and Ajani JA: Response to preoperative therapy in upper gastrointestinal cancers. Ann Surg Oncol 16: 878-886, 2009.

5. Morita M, Yoshida R, Ikeda K, Egashira A, Oki E, Sadanaga N, Kakeji Y, Yamanaka T and Maehara Y: Advances in esophageal cancer surgery in Japan: An analysis of 1000 consecutive patients treated at a single institute. Surgery 143: 499-508, 2008.

6. Allemani C, Matsuda T, Di Carlo V, Harewood R, Matz M, Nikšić M, Bonaventure A, Valkov M, Johnson CJ, Estève J, et al: Global surveillance of trends in cancer survival 2000-14 (CONCORD-3): Analysis of individual records for 37513 025 patients diagnosed with one of 18 cancers from 322 population-based registries in 71 countries. Lancet 391: 1023-1075, 2018.

7. Millard CJ, Fairall L and Schwabe JW: Towards an understanding of the structure and function of MTA1. Cancer Metastasis Rev 33: 857-867, 2014

8. Wu H, Moshkina N, Min J, Zeng H, Joshua J, Zhou MM and Plotnikov AN: Structural basis for substrate specificity and catalysis of human histone acetyltransferase 1. Proc Natl Acad Sci USA 109: 8925-8930, 2012.

9. Millard CJ, Varma N, Saleh A, Morris K, Watson PJ, Bottrill AR, Fairall L, Smith CJ and Schwabe JW: The structure of the core NuRD repression complex provides insights into its interaction with chromatin. Elife 5: e13941, 2016.

10. Christov CP, Dingwell KS, Skehel M, Wilkes HS, Sale JE, Smith JC and Krude T: A NuRD complex from xenopus laevis eggs is essential for DNA replication during early embryogenesis. Cell Rep 22: 2265-2278, 2018.

11. Xin J, Li J, Feng Y, Wang L, Zhang Y and Yang R: Downregulation of long noncoding RNA HOTAIRM1 promotes monocyte/dendritic cell differentiation through competitively binding to endogenous miR-3960. Onco Targets Ther 10: 1307-1315, 2017.

12. Balboula AZ, Stein P, Schultz RM and Schindler K: Knockdown of RBBP7 unveils a requirement of histone deacetylation for CPC function in mouse oocytes. Cell Cycle 13: 600-611, 2014.

13. Giri R, Yeh $\mathrm{HH}$, Wu CH and Liu HS: SUMO-1 overexpression increases RbAp46 protein stability and suppresses cell growth. Anticancer Res 28: 3749-3756, 2008.

14. Chen GC, Guan LS, Yu JH, Li GC, Choi Kim HR and Wang ZY: $\mathrm{Rb}$-associated protein 46 ( $\mathrm{RbAp} 46)$ inhibits transcriptional transactivation mediated by BRCA1. Biochem Biophys Res Commun 284: 507-514, 2001.

15. Zhang TF, Yu SQ, Loggie BW and Wang ZY: Inducible expression of RbAp46 activates c-Jun NH2-terminal kinase-dependent apoptosis and suppresses progressive growth of tumor xenografts in nude mice. Anticancer Res 23: 4621-4627, 2003.

16. Li GC, Guan LS and Wang ZY: Overexpression of RbAp46 facilitates stress-induced apoptosis and suppresses tumorigenicity of neoplastigenic breast epithelial cells. Int J Cancer 105: 762-768, 2003.

17. Rico M, Mukherjee A, Konieczkowski M, Bruggeman LA, Miller RT, Khan S, Schelling JR and Sedor JR: WT1-interacting protein and ZO-1 translocate into podocyte nuclei after puromycin aminonucleoside treatment. Am J Physiol Renal Physiol 289: F431-F441, 2005.

18. Li GC and Wang ZY: Constitutive expression of RbAp46 induces epithelial-mesenchymal transition in mammary epithelial cells. Anticancer Res 26: 3555-3560, 2006.

19. Yeh HH, Tseng YF, Hsu YC, Lan SH, Wu SY, Raghavaraju G, Cheng DE, Lee YR, Chang TY, Chow NH, et al: Ras induces experimental lung metastasis through up-regulation of RbAp46 to suppress RECK promoter activity. BMC Cancer 15: 172, 2015.

20. Fu J, Qin L, He T, Qin J, Hong J, Wong J, Liao L and Xu J: The TWIST/Mi2/NuRD protein complex and its essential role in cancer metastasis. Cell Res 21: 275-289, 2011

This work is licensed under a Creative Commons Attribution-NonCommercial-NoDerivatives 4.0 International (CC BY-NC-ND 4.0) License. 